\title{
La capacidad del barorreflejo como índice de identificación de los pacientes de UCI preparados para el destete
}

\author{
P. Armañac ${ }^{*}, 1$ D. Hernando ${ }^{1}$, J. Lázaro ${ }^{1}$ C. de Haro $^{2}$, R. Magrans ${ }^{2}$, L. Sarlabous ${ }^{2}$, JF. \\ López-Aguilar ${ }^{2}$, P. Laguna ${ }^{1}$, E. Gil ${ }^{1}$, L. Blanch ${ }^{2}$, R. Bailón ${ }^{1}$ \\ ${ }^{1}$ Biomedical Signal Interpretation and Computational Simulation (BSICoS), Instituto de Investigación en Ingeniería de \\ Aragón (I3A) Universidad de Zaragoza, (España). (*) parmanac@unizar.es \\ ${ }^{2}$ Hospital Universitari Parc Taulí - BetterCare, Barcelona (España)
}

\section{Resumen}

Actualmente, la Prueba de Respiración Espontánea (SBT), indica si un paciente está listo para el destete de la ventilación mecánica. Sin embargo, en torno al $20 \%$ de los pacientes "preparados" para ser desintubados, realmente no lo estaban. En este trabajo, se ha estudiado la Capacidad del Barorreflejo durante la hora anterior a la SBT, y se ha visto que existen diferencias significativas entre los pacientes listos para el destete y los que realmente no lo estaban, sugiriendo que la predicción del éxito del destete puede ser mejorada con este nuevo índice.

\section{Introducción}

Los pacientes que ingresan en las Unidades de Cuidados Intensivos (UCI), necesitan el apoyo de la Ventilación Mecánica (MV), para mantener la respiración. El destete es el proceso de la liberación de la MV en las UCI. Actualmente, la Prueba de Respiración Espontánea (SBT), indica si un paciente está listo para el destete. Sin embargo, la predicción de éxito del destete es poco precisa, ya que en torno al $20 \%$ de los pacientes "preparados" para ser desintubados, realmente no lo están. El mayor problema es que, para estos pacientes, su probabilidad de mortalidad sube hasta un 50\% [1].

El Sistema Nervioso Autonómico (ANS) es el encargado del control cardiovascular. Regula el acoplo entre los cambios en la presión arterial sitólica (SAP), en la frecuencia cardíaca (HR), y en la respiración. Los índices derivados de la Sensibilidad del Barorreflejo (BRS) -obtenida en base a la presión arterial-, y la Variabilidad del Ritmo Cardíaco (HRV), ya han demostrado ser capaces de estimar la regulación del ANS [2,3], y estudios previos ya revelan una alta incidencia de disfunción autonómica en los pacientes reintubados [4]. Sin embargo, ningún trabajo ha investigado el papel del BRS en el destete y nadie ha buscado diferencias mirando los registros, sólo, antes del SBT, con el fin de mejorar el valor predictivo de los índices de destete.

Este trabajo tiene como objetivo evaluar la BRS, en la última hora antes del SBT y explorar si el BRS proporciona información adicional para mejorar la predicción de éxito del destete. La BRS se evalúa mediante el análisis espectral de HRV y SAP y, de forma adicional, se mediante la capacidad (C), del BRS, utilizando el Promediado con Rectificación en Fase Bivariada (BPRSA), entre HRV y SAP.

\section{Materiales y Métodos}

\section{Base de Datos}

Los pacientes fueron monitorizados en las UCI adscritas al Hospital Universitari Parc Tauli de Sabadell, durante la hora previa al SBT. Las señales fisiológicas se registraron utilizando BetterCare ${ }^{\circledR}$, y para este trabajo se usan las grabaciones de la derivación II del electrocardiograma (ECG), de la frecuencia respiratoria, $\mathrm{F}_{\mathrm{r}}$, y la presión arterial.

Los pacientes se clasifican de forma que, cuando el estado de salud de un paciente en la UCI mejora lo suficiente, se evalúa si esta listo para el destete mediante la SBT [1]. Para los pacientes que superaron la SBT, se les consideró preparados para el destete y se les clasifica en el Sgroup. Para los pacientes que no pasaron la SBT, se les consideró fracaso del destete, y son clasificados en el Fgroup. Se dispone de 9 pacientes en el Sgroup y 6 pacientes en el Fgroup.

\section{Estimación de la HRV}

A partir de la señal de ECG, se detectan los instantes de ocurrencia de las ondas R, y se corrigen ectópicos. El tiempo entre dos ondas $R$ consecutivas, sin ectópicos, define el intervalo normal-normal (NN). A partir de la serie NN, se calculan los índices temporales de la HRV: desviación estándar de los intervalos NN (SDNN) y la media cuadrática de la diferencia entre intervalos sucesivos (RMSSD). Además, el modelo de Modulación de la Frecuencia de Pulsos por Integración se utiliza para estimar los cambios que genera el ANS en el HR, para obtener la señal moduladora, $m(n)$. La Potencia de la $m(n)$ en las bandas de Baja Frecuencia (LF) y Alta Frecuencia (HF), centrada en la respiración, se utilizan para estimar el balance simpatovagal, $\mathrm{P}_{\mathrm{LF}}^{n}$. 


\section{Estimación de la SAP y Análisis del BRS}

A partir de la señal de presión arterial, se detecta el máximo para cada pulso cardiaco. Con ello se define, posteriormente, la serie de presión sistólica, $d_{\mathrm{SAP}}(\mathrm{n})$. En este momento, la BRS se puede calcular mediante el análisis espectral del HRV y la SAP [2,3]:

$$
\alpha_{\mathrm{B}}(n)=\sqrt{\frac{\int_{\Omega_{\mathrm{B}}} \hat{S}_{\mathrm{HRV}}(n, f) d f}{\int_{\Omega_{\mathrm{B}}} \hat{S}_{\mathrm{SAP}}(n, f) d f}}, B \in\{L F, H F\},
$$

Las densidades espectrales de potencia, variantes en el tiempo, de la $m(n)$ y la $d_{\mathrm{SAP}}(n)$, se calculan mediante la distribución Tiempo-Frecuencia Wigner Ville, de la Clase de Cohen, con los parámetros ajustados para una resolución de $11.25 \mathrm{~s}$ en tiempo y $0.039 \mathrm{~Hz}$ en frecuencia.

\section{Análisis BPRSA}

Existe un método alternativo para analizar la función de la BRS, basado en el BPRSA [5]. En esencia, se obtiene la respuesta cardíaca general a aumentos en la SAP, calculando un perfil promedio del HRV. La BRS, estimada a partir de la curva BPRSA, se cuantifica con el índice de Capacidad, $C$ :

$$
C=\frac{1}{2 s} \sum_{i=1}^{s} \operatorname{BPRSA}(L+i)-\frac{1}{2 s} \sum_{i=0}^{s-1} \operatorname{BPRSA}(L-i) \text {. }
$$

La $C$, medida en $[\mathrm{ms}$ ], se basa en la wavelet de Haar, evaluada en una escala, $s$, y ubicada en $(L+1)$, en el centro de la curva BPRSA, de longitud $L$.

\section{Resultados y Discusión}

En la Tabla 1 se muestran todos los parámetros promediados en 1 hora. Se comparan los resultados obtenidos para el Sgroup y el Fgroup. La prueba Mann-Whitney se utiliza para el análisis estadístico. La capacidad, $C$, es el único índice que muestra diferencias significativas. La SDNN y RMSSD de la HRV, aunque no haya diferencias estadísticas, son notablemente más altas para el Sgroup. De hecho, otros trabajos sí encontraron, al comparar valores media hora antes del SBT y media hora después [4].

Tabla 1. Mediana [Q1,Q3] intersujeto, promediada en la hora antes del SBT, para todos los índices.

\begin{tabular}{l|ll|l} 
& Sgroup $(N=9)$ & Fgroup $(N=6)$ & $p$ \\
\hline HR $[\mathrm{bpm}]$ & $80[75,89]$ & $84[81,123]$ & 0.33 \\
$\mathrm{SAP}[\mathrm{mmHg}]$ & $132[110,139]$ & $126[101,141]$ & 0.78 \\
$\mathrm{~F}_{\mathrm{r}}[\mathrm{rpm}]$ & $19[18,23]$ & $22[18,24]$ & 0.61 \\
\hline $\mathrm{SDNN}[\mathrm{ms}]$ & $32[27,53]$ & $22[15,26]$ & 0.11 \\
$\mathrm{RMSSD}[\mathrm{ms}]$ & $11[6,14]$ & $5[3,8]$ & 0.18 \\
$\mathrm{P}_{\mathrm{LF}}^{n}$ & $0.56[0.3,0.65]$ & $0.66[0.41,0.8]$ & 0.39 \\
\hline$\alpha_{\mathrm{LF}}[\mathrm{ms} / \mathrm{mmHg}]$ & $6.0[3.5,11.8]$ & $5.1[2.3,7.1]$ & 0.46 \\
$\alpha_{\mathrm{HF}}[\mathrm{ms} / \mathrm{mmHg}]$ & $7.1[3.1,8.7]$ & $6.7[1.6,8.6]$ & 0.78 \\
$C[\mathrm{~ms}]$ & $-1.7[-2.7,-0.8]$ & $0.9[0.5,1.4]$ & $\mathbf{0 . 0 2}$
\end{tabular}

Como no se pueden extraer conclusiones sólidas de los valores promedio en toda la hora, se calcula la evolución en periodos promedio de 5 minutos. Los resultados se muestran en la Figura 1. Vuelven a ser visibles y significativas las diferencias en la $C$ y no se ven, tampoco, diferencias en los demás parámetros. Es importante señalar que la $C$, en valores absolutos, es mayor para el Sgroup, lo que podría deberse al efecto de la fuerza de los cambios de la SAP en la HRV. Además, se observa que la $C$ es negativa para el Sgroup pero positiva para el Fgroup, tal vez, ya que la actividad simpática se asocia con cambios de latencia más altos en la respuesta de la HRV a los aumentos espontáneos de la SAP.

\section{Conclusiones}

Se ha evaluado la BRS durante la hora previa al destete, en pacientes de UCI ventilados con ayuda de un respirador. La $C$ de la BRS, es mayor y negativa para los pacientes que estaban realmente listos para el destete. Estos resultados sugieren que la BRS es potencialmente útil para predecir los resultados del destete en la UCI y estudios futuros darán información útil para reducir las tasas de fracaso del destete y los importantes efectos adversos asociados.

\section{Referencias}

[1] BOLES JM, et al. Weaning from mechanical ventilation. Eur Resp J. 2007;29(5).

[2] LA ROVERE MT, et al. Baroreflex sensitivity: measurement and clinical implications. Ann NInv Electrocard 2008;13(2).

[3] PINNA GD, et al. Different estimation methods of spontaneous baroreflex sensitivity have different predictive value in heart failure patients. J Hypertension. 2017;35(8).

[4] CHEN YJ, et al. Vagal withdrawal and psychological distress during ventilator weaning and the related outcomes. $J$ Psychosom R. 2017;101.

[5] MÜLLER A, et al. Bivariate phase-rectified signal averaging for assessment of spontaneous baroreflex sensitivity: normalization of the results. J Electrocard. 2012;45(1).

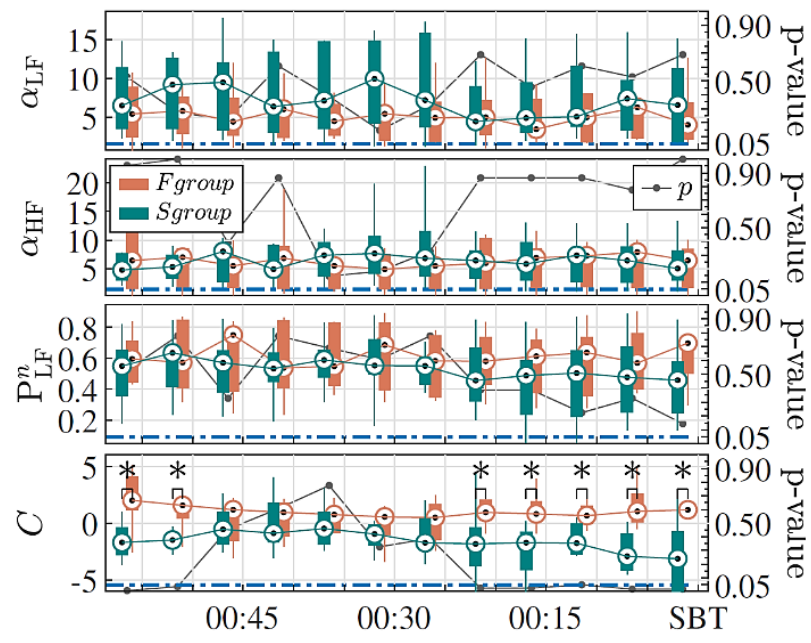

Figura 1. Evolución de los índices durante la hora previa al SBT. El BRS se mide con $\alpha$ en las bandas LF y $\mathrm{HF}[m s / m m H g]$ y con $C$ [ms]. La evolución de la HRV se evalúa mediante la $\mathbf{P}_{\mathbf{L F}}^{n}$.

Revista “Jornada de Jóvenes Investigadores del I3A”, vol. 8 (Actas de la IX Jornada de Jóvenes Investigadores del I3A -11 de diciembre de 2020). ISSN 2341-4790. 